\title{
UNIVALENCE AND CONVEXITY IN ONE DIRECTION OF THE CONVOLUTION OF HARMONIC MAPPINGS
}

\author{
SUMIT NAGPAL AND V. RAVICHANDRAN
}

\begin{abstract}
Let $\mathcal{H}$ denote the class of all complex-valued harmonic functions $f$ in the open unit disk normalized by $f(0)=0=f_{z}(0)-1=f_{\bar{z}}(0)$, and let $\mathcal{A}$ be the subclass of $\mathcal{H}$ consisting of normalized analytic functions. For $\phi \in \mathcal{A}$, let $\mathcal{W}_{H}^{-}(\phi):=\{f=h+\bar{g} \in$ $\mathcal{H}: h-g=\phi\}$ and $\mathcal{W}_{H}^{+}(\phi):=\{f=h+\bar{g} \in \mathcal{H}: h+g=\phi\}$ be subfamilies of $\mathcal{H}$. In this paper, we shall determine the conditions under which the harmonic convolution $f_{1} * f_{2}$ is univalent and convex in one direction if $f_{1} \in \mathcal{W}_{H}^{-}(z)$ and $f_{2} \in \mathcal{W}_{H}^{-}(\phi)$. A similar analysis is carried out if $f_{1} \in \mathcal{W}_{H}^{-}(z)$ and $f_{2} \in \mathcal{W}_{H}^{+}(\phi)$. Examples of univalent harmonic mappings constructed by way of convolution are also presented.
\end{abstract}

\section{INTRODUCTION}

Let $\mathcal{H}$ denote the class of all complex-valued harmonic functions $f$ in the open unit disk $\mathbb{D}=\{z \in \mathbb{C}:|z|<1\}$ normalized by $f(0)=0=f_{z}(0)-1=f_{\bar{z}}(0)$. Such functions can be written in the form $f=h+\bar{g}$, where

$$
h(z)=z+\sum_{n=2}^{\infty} a_{n} z^{n} \quad \text { and } \quad g(z)=\sum_{n=2}^{\infty} b_{n} z^{n}
$$

are analytic in $\mathbb{D}$. The quantity $w_{f}=g^{\prime} / h^{\prime}$ is called the dilatation of a harmonic mapping $f$. A function $f \in \mathcal{H}$ is sense-preserving in $\mathbb{D}$ if the Jacobian $J_{f}=\left|h^{\prime}\right|^{2}-\left|g^{\prime}\right|^{2}$ is positive or equivalently $\left|g^{\prime}\right|<\left|h^{\prime}\right|$ in $\mathbb{D}$. The dilatation $w_{f}$ of a sense-preserving harmonic mapping $f$ is analytic and satisfies $\left|w_{f}\right|<1$ in $\mathbb{D}$. Let $\mathcal{S}_{H}^{0}$ be the subclass of $\mathcal{H}$ consisting of univalent and sense-preserving functions. The classical family $\mathcal{S}$ of normalized analytic univalent functions is a subclass of $\mathcal{S}_{H}^{0}$. Finally, let $\mathcal{K}$ and $\mathcal{K}_{H}^{0}$ be the subclasses of $\mathcal{S}$ and $\mathcal{S}_{H}^{0}$ respectively, mapping $\mathbb{D}$ onto a convex domain.

Clunie and Sheil-Small [1] introduced the method of "shear construction" to produce a harmonic mapping with a specified dilatation onto a domain convex in one direction by shearing a given conformal mapping along parallel lines. A domain $\Omega \subset \mathbb{C}$ is convex in the direction of real (resp. imaginary) axis if its intersection with each horizontal (resp. vertical) line is connected. A function $f \in \mathcal{H}$ is convex in the direction of real (resp. imaginary) axis if it maps $\mathbb{D}$ onto a domain convex in the direction of real (resp. imaginary) axis. The shear construction is contained in the following lemma.

Lemma 1.1. [1] A sense-preserving harmonic function $f=h+\bar{g}$ in $\mathbb{D}$ is a univalent mapping of $\mathbb{D}$ convex in the direction of real (resp. imaginary) axis if and only if $h-g$

1991 Mathematics Subject Classification. Primary 31A05; Secondary 30C45.

Key words and phrases. univalent harmonic mappings; sense-preserving; convex in one direction; convolution. 
(resp. $h+g)$ is a conformal univalent mapping of $\mathbb{D}$ convex in the direction of the real (resp. imaginary) axis.

For analytic functions $\phi(z)=z+\sum_{n=2}^{\infty} a_{n} z^{n}$ and $\psi(z)=z+\sum_{n=2}^{\infty} A_{n} z^{n}$, their convolution (or Hadamard product) is defined as $(\phi * \psi)(z)=z+\sum_{n=2}^{\infty} a_{n} A_{n} z^{n}, z \in \mathbb{D}$. In the harmonic case, with $f=h+\bar{g}$ and $F=H+\bar{G}$, their harmonic convolution is defined as $f * F=h * H+\overline{g * G}$. The right half-plane mapping $l(z)=z /(1-z)$ act as convolution identity for analytic functions, while the function $e(z)=z /(1-z)+\bar{z}^{2} /(1-\bar{z}) \in \mathcal{H}$ is an identity under the harmonic convolutions. Note that $e \notin \mathcal{S}_{H}^{0}$. Harmonic convolutions are investigated in [1, 2, 3, 4, 7, 11, 12].

Let $\mathcal{A}$ be the subclass of $\mathcal{H}$ consisting of normalized analytic functions. For $\phi \in \mathcal{A}$, consider the following subfamilies of $\mathcal{H}$ :

$$
\mathcal{W}_{H}^{-}(\phi):=\{f=h+\bar{g} \in \mathcal{H}: h-g=\phi\},
$$

and

$$
\mathcal{W}_{H}^{+}(\phi):=\{f=h+\bar{g} \in \mathcal{H}: h+g=\phi\} .
$$

Dorff [2] proved that if $f_{1}, f_{2} \in \mathcal{W}_{H}^{+}(z /(1-z))$ and $f_{1}, f_{2}, f_{1} * f_{2}$ are all sense-preserving in $\mathbb{D}$ then $f_{1} * f_{2} \in \mathcal{S}_{H}^{0}$ and is convex in the direction of real axis. Dorff et al. [3] established the cases under which the assumption of $f_{1} * f_{2}$ to be sense-preserving can be omitted. In this paper, we shall investigate the convolution properties of the classes $\mathcal{W}_{H}^{-}(\phi)$ and $\mathcal{W}_{H}^{+}(\phi)$, for specific choices of $\phi$.

In Section 2, we shall determine the conditions under which the harmonic convolution $f_{1} * f_{2}$ is univalent and convex in one direction if $f_{1} \in \mathcal{W}_{H}^{-}(z)$ and $f_{2} \in \mathcal{W}_{H}^{-}(\phi)$. Although the functions in these classes need not be univalent in $\mathbb{D}$, but it has been shown that their convolution is univalent in $\mathbb{D}$ and convex in the direction of real axis, under certain milder conditions. It turns out to be a simple, but an effective tool in the construction of univalent harmonic mappings convex in a given direction.

A similar analysis is carried out in Section 3 to determine the conditions for the harmonic convolution $f_{1} * f_{2}$ to be univalent and convex in the direction of imaginary axis if $f_{1} \in \mathcal{W}_{H}^{-}(z)$ and $f_{2} \in \mathcal{W}_{H}^{+}(\phi)$.

\section{UNIVAlEnCE AND CONVEXITy IN THE DIRECTION OF REAL AXIS}

The first theorem of this section gives a sufficient condition for univalence and convexity in the direction of real axis of the harmonic convolution $f_{1} * f_{2}$ if $f_{1} \in \mathcal{W}_{H}^{-}(z)$ and $f_{2} \in \mathcal{W}_{H}^{-}(\phi)$.

Theorem 2.1. Let $f_{1}=h_{1}+\bar{g}_{1} \in \mathcal{W}_{H}^{-}(z)$ and $f_{2}=h_{2}+\bar{g}_{2} \in \mathcal{W}_{H}^{-}(\phi)$. Then

(i) $f_{1} * f_{2} \in \mathcal{W}_{H}^{-}\left(h_{1} * \phi\right)$;

(ii) If the analytic function $h_{1} * \phi$ is univalent and convex in the direction of real axis and

$$
\operatorname{Re} \frac{\left(h_{1} * h_{2}\right)^{\prime}(z)}{\left(h_{1} * \phi\right)^{\prime}(z)}>\frac{1}{2}, \quad z \in \mathbb{D}
$$

then $f_{1} * f_{2} \in S_{H}^{0}$ and is convex in the direction of real axis. 
Proof. Recall that $\psi * z=z=z * \psi$ for any analytic function $\psi$ with $\psi^{\prime}(0)=1$, so we can write

$$
\begin{aligned}
z=z *\left(h_{2}+g_{2}\right) & =\left(h_{1}-g_{1}\right) *\left(h_{2}+g_{2}\right) \\
& =\left(h_{1} * h_{2}\right)+\left(h_{1} * g_{2}\right)-\left(h_{2} * g_{1}\right)-\left(g_{1} * g_{2}\right)
\end{aligned}
$$

and

$$
\begin{aligned}
\left(h_{1}+g_{1}\right) * \phi & =\left(h_{1}+g_{1}\right) *\left(h_{2}-g_{2}\right) \\
& =\left(h_{1} * h_{2}\right)-\left(h_{1} * g_{2}\right)+\left(h_{2} * g_{1}\right)-\left(g_{1} * g_{2}\right) .
\end{aligned}
$$

Thus, we obtain

$$
\begin{aligned}
\left(h_{1} * h_{2}\right)-\left(g_{1} * g_{2}\right) & =\frac{1}{2}\left[z+\left(h_{1}+g_{1}\right) * \phi\right] \\
& =\frac{1}{2}\left[z+2\left(h_{1} * \phi\right)-z * \phi\right]=h_{1} * \phi,
\end{aligned}
$$

since $h_{1}-g_{1}=z$. This proves (i).

For the proof of (ii), note that $\left(h_{1} * h_{2}\right)-\left(g_{1} * g_{2}\right)$ is univalent and convex in the direction of real axis. In order to apply Lemma 1.1 to the function $f_{1} * f_{2}$, we need to show that the dilatation $w_{f_{1} * f_{2}}=\left(g_{1} * g_{2}\right)^{\prime} /\left(h_{1} * h_{2}\right)^{\prime}$ of $f_{1} * f_{2}$ satisfies $\left|w_{f_{1} * f_{2}}(z)\right|<1$ for all $z \in \mathbb{D}$. Equivalently, it suffices to show that $\operatorname{Re}\left(\left(1+w_{f_{1} * f_{2}}\right) /\left(1-w_{f_{1} * f_{2}}\right)\right)>0$ in $\mathbb{D}$. Using the identity $h_{1} * \phi=\left(h_{1} * h_{2}\right)-\left(g_{1} * g_{2}\right)$, we see that

$$
\begin{aligned}
\operatorname{Re}\left(\frac{1+w_{f_{1} * f_{2}}}{1-w_{f_{1} * f_{2}}}\right) & =\operatorname{Re} \frac{\left(h_{1} * h_{2}\right)^{\prime}+\left(g_{1} * g_{2}\right)^{\prime}}{\left(h_{1} * h_{2}\right)^{\prime}-\left(g_{1} * g_{2}\right)^{\prime}} \\
& =\operatorname{Re} \frac{\left(h_{1} * h_{2}\right)^{\prime}+\left(g_{1} * g_{2}\right)^{\prime}}{\left(h_{1} * \phi\right)^{\prime}} \\
& =2 \operatorname{Re} \frac{\left(h_{1} * h_{2}\right)^{\prime}}{\left(h_{1} * \phi\right)^{\prime}}-1
\end{aligned}
$$

which is clearly positive, under the hypothesis of the theorem.

Making use of Theorem 2.1, we will investigate the convolution properties of functions in the class $\mathcal{W}_{H}^{-}(z)$. Taking $\phi(z) \equiv z$, we obtain

Corollary 2.2. Let both $f_{1}=h_{1}+\bar{g}_{1}, f_{2}=h_{2}+\bar{g}_{2} \in \mathcal{W}_{H}^{-}(z)$. Then

(i) $f_{1} * f_{2} \in \mathcal{W}_{H}^{-}(z)$. In particular, the class $\mathcal{W}_{H}^{-}(z)$ is closed under harmonic convolution:

(ii) If $\operatorname{Re}\left(h_{1} * h_{2}\right)^{\prime}>1 / 2$ in $\mathbb{D}$, then $f_{1} * f_{2} \in S_{H}^{0}$ and is convex in the direction of real axis.

It is easy to see that the range of $f \in \mathcal{W}_{H}^{-}(z)$ is contained in the horizontal strip $|\operatorname{Im} w|<1$. Corollary 2.2 shows that the same is true for the harmonic convolution $f_{1} * f_{2}$ if $f_{1}, f_{2} \in \mathcal{W}_{H}^{-}(z)$. Now, we provide some examples to illustrate Corollary 2.2 ,

Example 2.3. For $n=2,3, \ldots$, let $p_{n}=u_{n}+\bar{v}_{n}$ be the harmonic mappings of $\mathbb{D}$ with $u_{n}=z+z^{n} / n$ and $v_{n}=z^{n} / n$. Then $p_{n} \in \mathcal{W}_{H}^{-}(z)$ and

$$
p_{n} * p_{n}=u_{n} * u_{n}+\overline{v_{n} * v_{n}}=z+\frac{z^{n}}{n^{2}}+\frac{\bar{z}^{n}}{n^{2}}
$$


for $n=2,3, \ldots$ For $z \in \mathbb{D}, \operatorname{Re}\left(u_{n} * u_{n}\right)^{\prime}(z)=1+\operatorname{Re} z^{n-1} / n>1-1 / n \geq 1 / 2$ which imply that the convolution maps $p_{n} * p_{n}(n=2,3, \ldots)$ are univalent and convex in the direction of real axis, by Corollary 2.2. The images of the unit disk under $p_{n}$ and $p_{n} * p_{n}$ for $2 \leq n \leq 4$ are shown in Figure 1 as plots of the images of equally spaced radial segments and concentric circles.

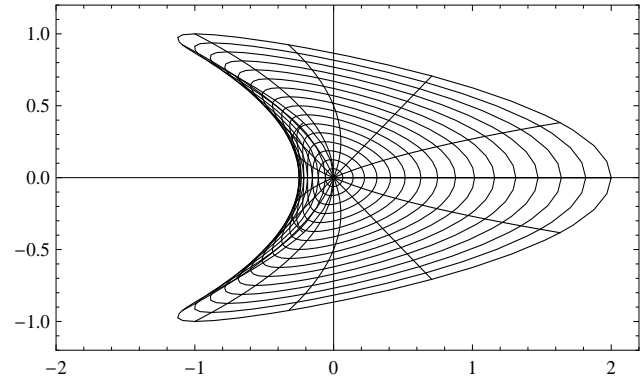

(a) $p_{2}$

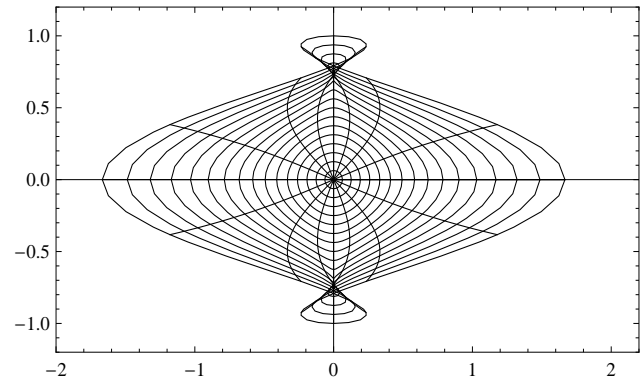

(c) $p_{3}$

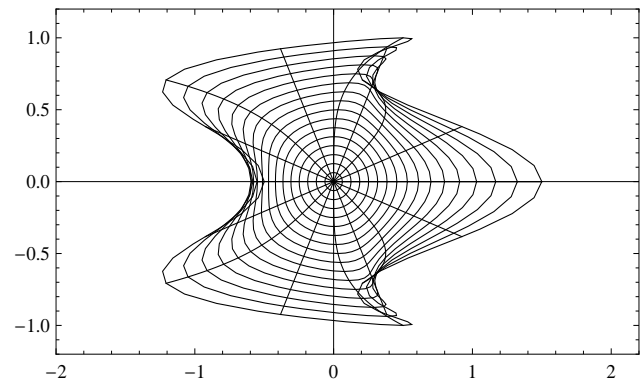

(e) $p_{4}$

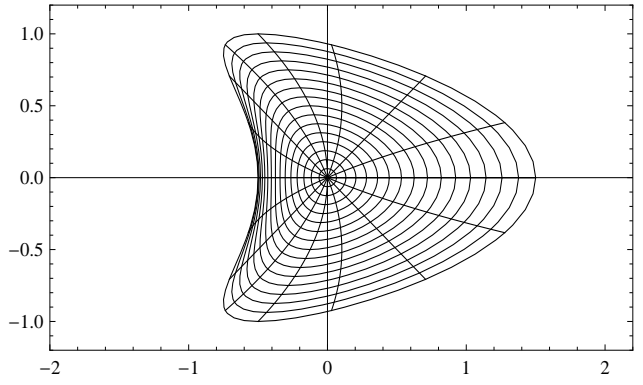

(b) $p_{2} * p_{2}$

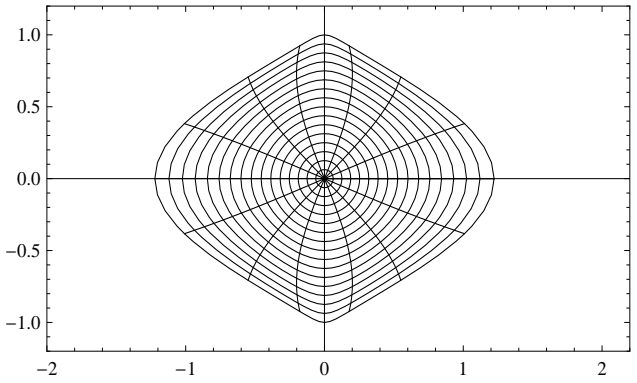

(d) $p_{3} * p_{3}$

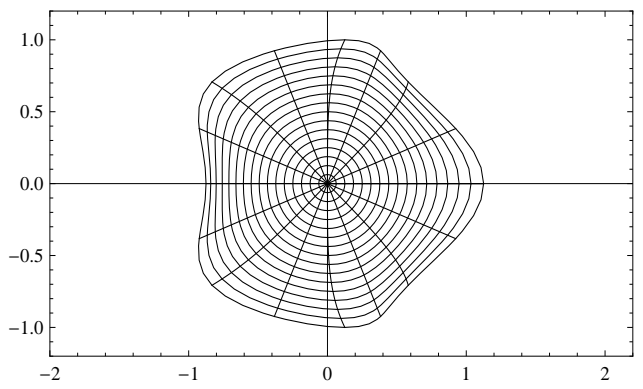

(f) $p_{4} * p_{4}$

Figure 1. Images of the functions $p_{n}(z)=z+z^{n} / n+\bar{z}^{n} / n$ and their convolutions $p_{n} * p_{n}$ for $n=2,3,4$.

Note that the mappings $p_{n} \in \mathcal{W}_{H}^{-}(z)(n=2,3, \ldots)$ are not univalent in $\mathbb{D}$ since their Jacobian $J_{p_{n}}(z)=1+2 \operatorname{Re} z^{n-1}$ vanishes inside $\mathbb{D}$.

Remark 2.4. The images of the convolution maps $p_{n} * p_{n}$ (see Figure 11) in Example 2.3 suggest that $p_{n} * p_{n} \notin \mathcal{K}_{H}^{0}$. Thus, the conclusion of the Corollary 2.2 can't to strengthened to $f_{1} * f_{2} \in \mathcal{K}_{H}^{0}$. 
Example 2.5. For $k=1,2, \ldots$, let $\Gamma_{k}=\mu_{k}+\bar{\nu}_{k}$ be the shears of the identity map in the direction of real axis with dilatation $w_{\Gamma_{k}}(z)=z^{k}$. Then $\Gamma_{k} \in \mathcal{W}_{H}^{-}(z)$ and

$$
\mu_{k}(z)=z+\sum_{n=1}^{\infty} \frac{z^{n k+1}}{n k+1}, \quad \nu_{k}(z)=\sum_{n=1}^{\infty} \frac{z^{n k+1}}{n k+1} \quad(z \in \mathbb{D} ; k=1,2, \ldots) .
$$

In particular, we have

$$
\Gamma_{1}=\mu_{1}+\bar{\nu}_{1}, \quad \mu_{1}(z)=-\log (1-z), \quad \nu_{1}(z)=-z-\log (1-z)
$$

and

$$
\Gamma_{2}=\mu_{2}+\bar{\nu}_{2}, \quad \mu_{2}(z)=\frac{1}{2} \log \left(\frac{1+z}{1-z}\right), \quad \nu_{2}(z)=-z+\frac{1}{2} \log \left(\frac{1+z}{1-z}\right)
$$

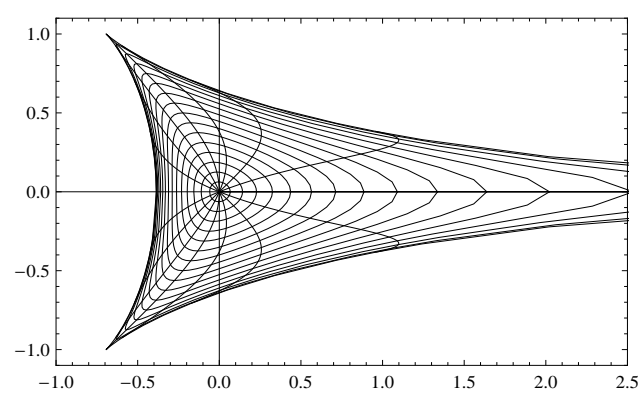

(a) $\Gamma_{1}$

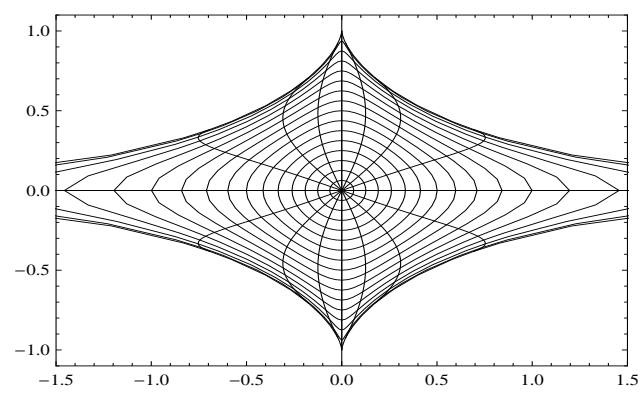

(c) $\Gamma_{2}$

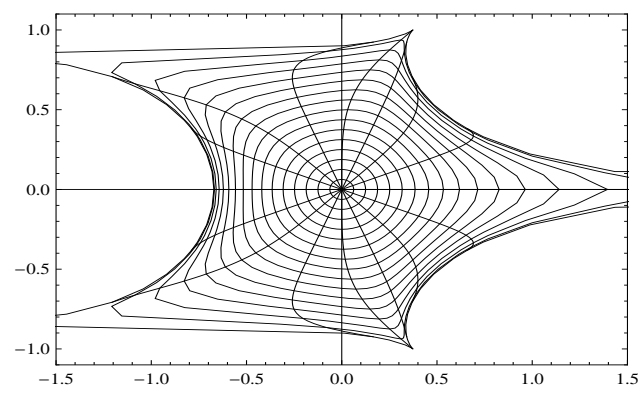

(e) $\Gamma_{3}$

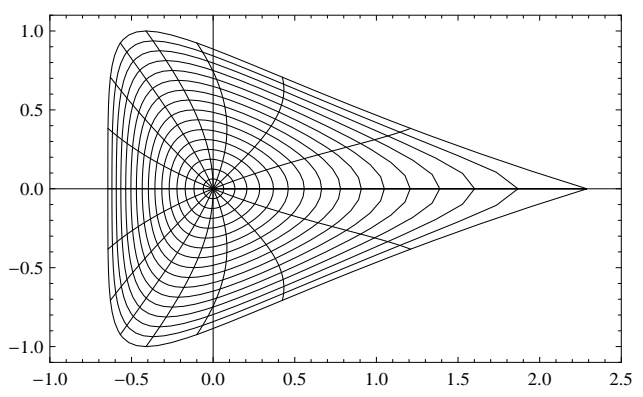

(b) $\Gamma_{1} * \Gamma_{1}$

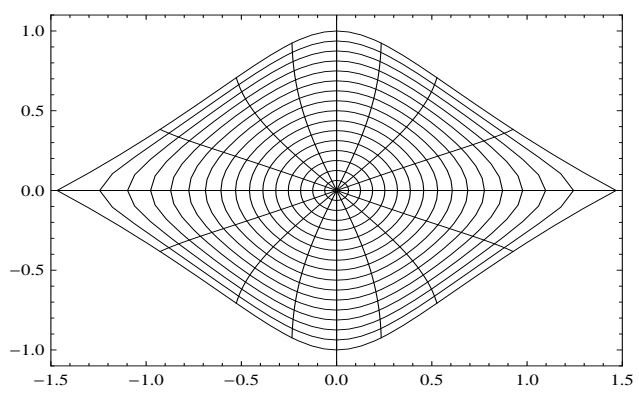

(d) $\Gamma_{2} * \Gamma_{2}$

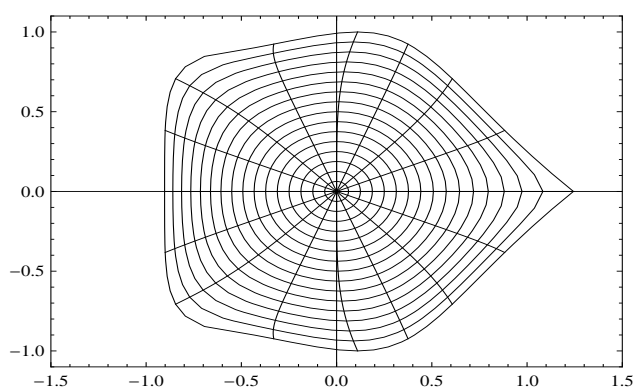

(f) $\Gamma_{3} * \Gamma_{3}$

Figure 2. Images of the functions $\Gamma_{k}$ and their convolutions $\Gamma_{k} * \Gamma_{k}$ for $k=1,2,3$. 
To apply Corollary 2.2 for the functions $\Gamma_{k} * \Gamma_{k}$, we need to show that $\operatorname{Re}\left(\mu_{k} * \mu_{k}\right)^{\prime}>1 / 2$ $(k=1,2, \ldots)$ in $\mathbb{D}$. Note that

$$
\left(\mu_{k} * \mu_{k}\right)(z)=z+\sum_{n=1}^{\infty} \frac{z^{n k+1}}{(n k+1)^{2}}, \quad z \in \mathbb{D} .
$$

Differentiation with respect to $z$ leads to an expression

$$
z\left(\mu_{k} * \mu_{k}\right)^{\prime \prime}(z)+\left(\mu_{k} * \mu_{k}\right)^{\prime}(z)=1+\sum_{n=1}^{\infty} z^{n k}=\frac{1}{1-z^{k}}, \quad z \in \mathbb{D} .
$$

This shows that

$$
\operatorname{Re}\left(z\left(\mu_{k} * \mu_{k}\right)^{\prime \prime}(z)+\left(\mu_{k} * \mu_{k}\right)^{\prime}(z)\right)>1 / 2, \quad z \in \mathbb{D} .
$$

By [13, Theorem 2], it follows that $\operatorname{Re}\left(\mu_{k} * \mu_{k}\right)^{\prime}>\log 2 \simeq 0.69314$ in $\mathbb{D}$. Now, Corollary 2.2 shows that the convolution maps

$$
\left(\Gamma_{k} * \Gamma_{k}\right)(z)=z+\sum_{n=1}^{\infty} \frac{z^{n k+1}}{(n k+1)^{2}}+\overline{\sum_{n=1}^{\infty} \frac{z^{n k+1}}{(n k+1)^{2}}} \quad(z \in \mathbb{D}, k=1,2, \ldots)
$$

are univalent and convex in the direction of real axis. Figure 2 depicts the image domains $\Gamma_{k}(\mathbb{D})$ and $\left(\Gamma_{k} * \Gamma_{k}\right)(\mathbb{D})$ for $k=1,2,3$.

The next theorem deals with the convolution of $\Gamma_{1} \in \mathcal{W}_{H}^{-}(z)$ given by (2) with harmonic mappings $f \in \mathcal{W}_{H}^{-}(\phi)$.

Theorem 2.6. Let $f=h+\bar{g} \in \mathcal{W}_{H}^{-}(\phi)$ with $\operatorname{Re} h(z) / \phi(z)>1 / 2$ for all $z \in \mathbb{D}$. If the analytic function $\mu_{1} * \phi$ is univalent and convex in the direction of real axis, then $\Gamma_{1} * f \in \mathcal{S}_{H}^{0}$ and is convex in the direction of real axis, $\Gamma_{1}=\mu_{1}+\bar{\nu}_{1}$ is given by (2).

Proof. Note that $z\left(\mu_{1} * h\right)^{\prime}=z \mu_{1}^{\prime} * h=z /(1-z) * h=h$. Similarly, $z\left(\mu_{1} * \phi\right)^{\prime}=\phi$. Therefore $\operatorname{Re}\left(\mu_{1} * h\right)^{\prime} /(\phi * h)^{\prime}=\operatorname{Re} h / \phi>1 / 2$ in $\mathbb{D}$. The result now follows by applying Theorem 2.1.

Example 2.7. Consider the non-univalent harmonic function $f=h+\bar{g}$ where $h(z)=$ $z(1+z) /(1-z)^{2}$ and $g(z)=z^{2}(1+z) /(1-z)^{2}$. Then $f \in \mathcal{W}_{H}^{-}(\phi), \phi(z)=z(1+z) /(1-z)$. Note that $\operatorname{Re} h(z) / \phi(z)=\operatorname{Re} 1 /(1-z)>1 / 2$ and $\left(\mu_{1} * \phi\right)(z)=-z-2 \log (1-z)$ is univalent and convex in the direction of real axis. By Theorem [2.6, the convolution

$$
\left(\Gamma_{1} * f\right)(z)=\frac{2 z}{1-z}+\log (1-z)+\overline{\frac{3 z-z^{2}}{1-z}+3 \log (1-z)}, \quad z \in \mathbb{D} .
$$

is univalent and convex in the direction of real axis (see Figure 3).

In [9], the authors introduced the notion of positive harmonic Alexander operator $\Lambda_{H}^{+}$: $\mathcal{H} \rightarrow \mathcal{H}$ defined by

$$
\Lambda_{H}^{+}[f](z)=\int_{0}^{z} \frac{h(t)}{t} d t+\overline{\int_{0}^{z} \frac{g(t)}{t} d t}, \quad(f=h+\bar{g} \in \mathcal{H} ; z \in \mathbb{D}) .
$$

In general, $\Lambda_{H}^{+}\left[\mathcal{S}_{H}^{0}\right] \not \subset \mathcal{S}_{H}^{0}$. Since $\Lambda_{H}^{+}[f]=f * \Gamma_{1}$ where $\Gamma_{1}$ is given by (2), Theorem 2.6 determines a class of harmonic mappings that is mapped into $\mathcal{S}_{H}^{0}$ by the positive harmonic Alexander operator $\Lambda_{H}^{+}$. For specific choices of $\phi$ in Theorem 2.6, we obtain the following corollary. 


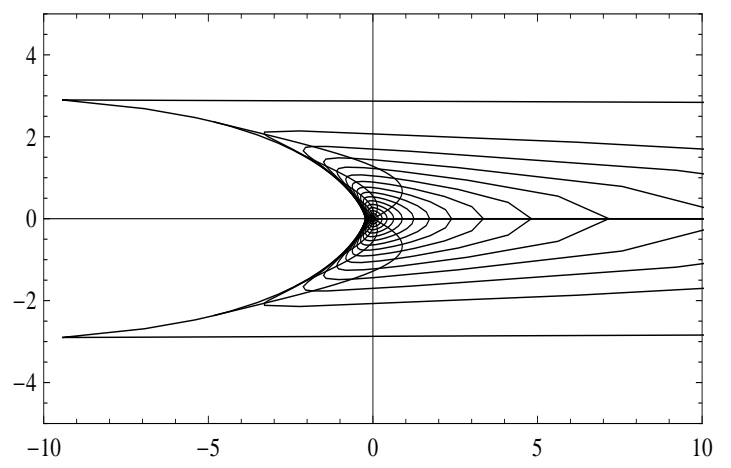

Figure 3. Image of the convolution of $\Gamma_{1}$ with $f(z)=z(1+z) /(1-z)^{2}+$ $\bar{z}^{2}(1+\bar{z}) /(1-\bar{z})^{2}$.

Corollary 2.8. Suppose that $f=h+\bar{g} \in \mathcal{H}$ satisfies one of the following three conditions:

(i) $f \in \mathcal{W}_{H}^{-}(z)$ and $\operatorname{Re} h(z) / z>1 / 2(z \in \mathbb{D})$;

(ii) $f \in \mathcal{W}_{H}^{-}(z /(1-z))$ and $\operatorname{Re}(1-z) h(z) / z>1 / 2(z \in \mathbb{D})$;

(iii) $f \in \mathcal{W}_{H}^{-}\left(z /(1-z)^{2}\right)$ and $\operatorname{Re}(1-z)^{2} h(z) / z>1 / 2(z \in \mathbb{D})$.

Then $\Lambda_{H}^{+}[f] \in \mathcal{S}_{H}^{0}$ and is convex in the direction of real axis.

Suppose that $f=h+\bar{g} \in \mathcal{K}_{H}^{0}$. Then $|h(z)|>|g(z)|$ for all $z \in \mathbb{D} \backslash\{0\}$ by 11, Corollary 5.8]. If, in addition, $h(z)-g(z)=z$ then $|h(z)|>|h(z)-z|$ which imply that $|h(z) / z|>$ $|h(z) / z-1|$ for all $z \in \mathbb{D} \backslash\{0\}$. This shows that $\operatorname{Re} h(z) / z>1 / 2$ for $z \in \mathbb{D} \backslash\{0\}$. The inequality $\operatorname{Re} h(z) / z>1 / 2$ is obviously true if $z=0$. Therefore Corollary 2.8(i) gives

Corollary 2.9. If $f \in \mathcal{W}_{H}^{-}(z) \cap \mathcal{K}_{H}^{0}$ then $\Lambda_{H}^{+}[f] \in S_{H}^{0}$ and is convex in the direction of real axis.

Example 2.10. The function $f(z)=z+z^{2} / 8+\bar{z}^{2} / 8 \in \mathcal{W}_{H}^{-}(z)$ is sense-preserving and satisfies

$$
\frac{\partial}{\partial \theta}\left(\arg \left\{\frac{\partial}{\partial \theta} f\left(r e^{i \theta}\right)\right\}\right)>0, \quad 0 \leq \theta<2 \pi, \quad 0<r<1 .
$$

This shows that $f$ is fully convex in $\mathbb{D}$ (see [6, 8] ) and hence $f \in \mathcal{K}_{H}^{0}$. By Corollary 2.9, the convolution $\left(\Gamma_{1} * f\right)(z)=z+z^{2} / 16+\bar{z}^{2} / 16$ is univalent in $\mathbb{D}$ and convex in the direction of real axis (see Figure 4).

Corollary 2.11. If $f=h+\bar{g} \in \mathcal{W}_{H}^{-}(z)$ with $h \in \mathcal{K}$, then $\Lambda_{H}^{+}[f] \in S_{H}^{0}$ and is convex in the direction of real axis.

Proof. Since $h \in \mathcal{K}$, Re $h(z) / z>1 / 2$ for $z \in \mathbb{D}$ by the well-known Marx Strohhäcker theorem [5, Theorem 2.6(a), p. 57]. By Corollary 2.8(i), we obtain the desired result.

Since $\mu_{1} \in \mathcal{K}$, Corollary 2.11 directly shows that the convolution $\Gamma_{1} * \Gamma_{1} \in \mathcal{S}_{H}^{0}$ and is convex in the direction of real axis, where $\Gamma_{1}=\mu_{1}+\bar{\nu}_{1} \in \mathcal{W}_{H}^{-}(z)$ is given by (2). Parts (ii) and (iii) of Corollary 2.8 are illustrated by the following examples.

Example 2.12. The harmonic mapping

$$
F(z)=U(z)+\overline{V(z)}, \quad U(z):=\frac{z-\frac{1}{2} z^{2}}{(1-z)^{2}}, \quad V(z):=\frac{\frac{1}{2} z^{2}}{(1-z)^{2}}
$$




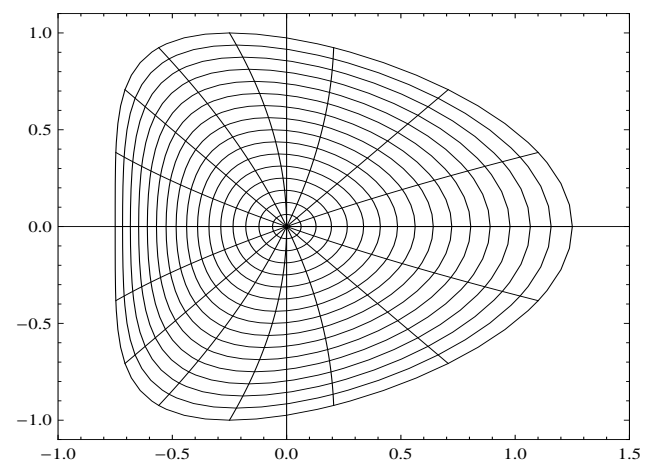

(a) $f$

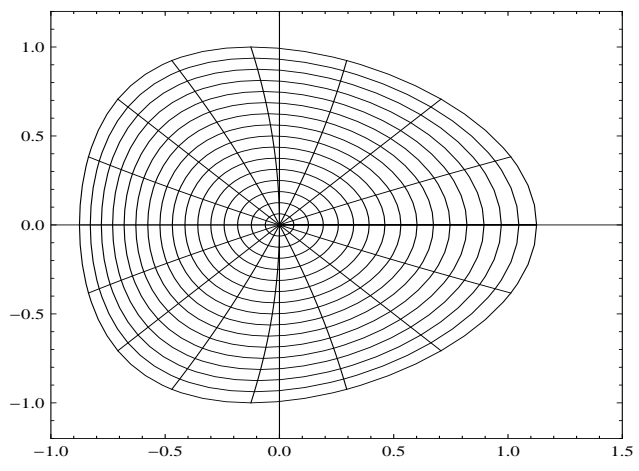

(b) $\Gamma_{1} * f$

Figure 4. Images of the function $f(z)=z+z^{2} / 8+\bar{z}^{2} / 8$ and its convolution with $\Gamma_{1}$.

constructed by shearing the conformal mapping $l(z)=z /(1-z)$ in the direction of real axis with dilatation $w_{F}(z)=z$, belongs to $\mathcal{W}_{H}^{-}(z /(1-z))$ and $F(\mathbb{D})=\left\{u+i v: v^{2}>-(u+1 / 4)\right\}$ (see Figure 5(a)). Recently, the authors [10] calculated the radius of starlikeness and convexity of the function $F$. Note that $\operatorname{Re}(1-z) U(z) / z=\operatorname{Re}(2-z) /(2(1-z))>3 / 4$ for all $z \in \mathbb{D}$. By Corollary 2.8(ii), the convolution

$$
\left(\Gamma_{1} * F\right)(z)=\operatorname{Re} \frac{z}{1-z}-i \arg \{1-z\}, \quad z \in \mathbb{D}
$$

is univalent and convex in the direction of real axis (see Figure 5(b)).

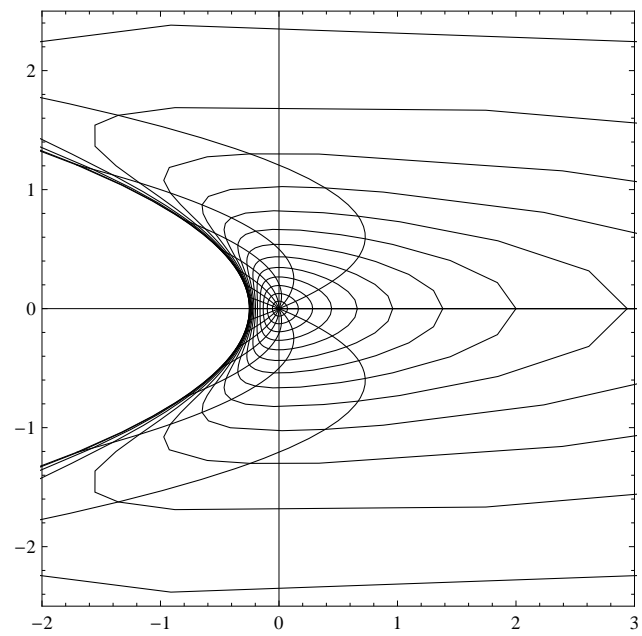

(a) $F$

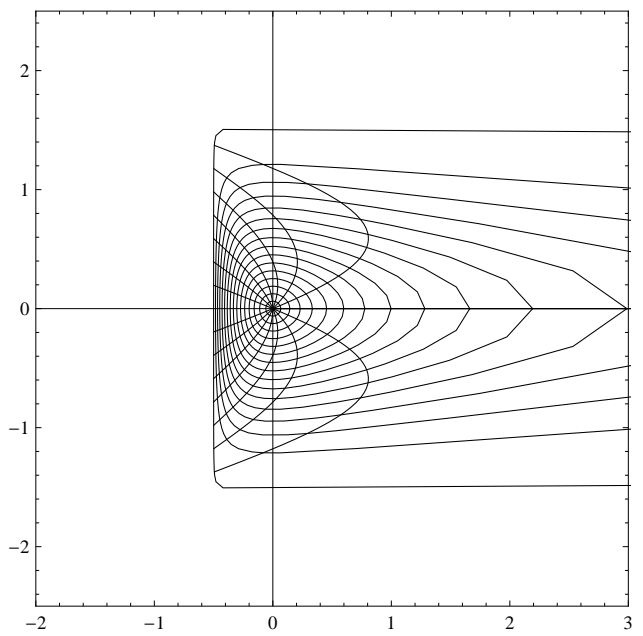

(b) $\Gamma_{1} * F$

FiguRE 5. Images of the function $F$ and its convolution with $\Gamma_{1}$

Example 2.13. The harmonic Koebe function

$$
K(z)=H(z)+\overline{G(z)}, \quad H(z):=\frac{z-\frac{1}{2} z^{2}+\frac{1}{6} z^{3}}{(1-z)^{3}}, \quad G(z):=\frac{\frac{1}{2} z^{2}+\frac{1}{6} z^{3}}{(1-z)^{3}}
$$




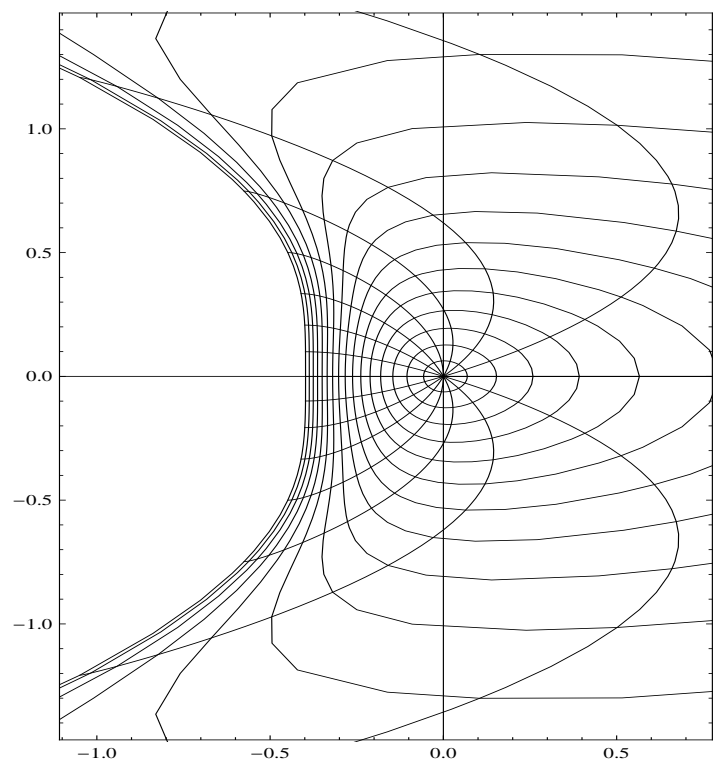

FiguRE 6 . Image of the convolution of $\Gamma_{1}$ with the harmonic Koebe function $K$.

constructed by shearing the Koebe function $k(z)=z /(1-z)^{2}$ in the direction of real axis with dilatation $w_{K}(z)=z$, maps the unit disk $\mathbb{D}$ onto the slit-plane $\mathbb{C} \backslash(-\infty,-1 / 6]$. Note that $K \in \mathcal{W}_{H}^{-}\left(z /(1-z)^{2}\right)$ and $\operatorname{Re}(1-z)^{2} H(z) / z>1 / 2$ so the convolution

$$
\Lambda_{H}^{+}[K]=\left(\Gamma_{1} * K\right)(z)=\frac{2}{3} \frac{z}{(1-z)^{2}}+\frac{1}{3} i \operatorname{Im} \frac{z-3 z^{2}}{(1-z)^{2}}-\frac{1}{3} \log |1-z|, \quad z \in \mathbb{D}
$$

is univalent and convex in the direction of real axis (see Figure 6) by Corollary 2.8(iii).

Analogous to Theorem 2.1, the last theorem of this section determines the conditions under which the harmonic convolution $f_{1} * f_{2}$ is univalent and convex in one direction if $f_{1} \in \mathcal{W}_{H}^{+}(z)$ and $f_{2} \in \mathcal{W}_{H}^{+}(\phi)$. Its proof follows by an easy modification of the proof of Theorem 2.1.

Theorem 2.14. Let $f_{1}=h_{1}+\bar{g}_{1} \in \mathcal{W}_{H}^{+}(z)$ and $f_{2}=h_{2}+\bar{g}_{2} \in \mathcal{W}_{H}^{+}(\phi)$. Then $f_{1} * f_{2} \in$ $\mathcal{W}_{H}^{-}\left(h_{1} * \phi\right)$. Moreover, if $h_{1} * \phi \in \mathcal{S}$ and is convex in the direction of real axis with $\operatorname{Re}\left(h_{1} * h_{2}\right)^{\prime} /\left(h_{1} * \phi\right)^{\prime}>1 / 2$ in $\mathbb{D}$ then $f_{1} * f_{2} \in S_{H}^{0}$ and is convex in the direction of real axis.

\section{Univalence AND CONVEXity IN THE DiRECTION OF IMAGINARY AXIS}

In this section, we shall investigate the properties of the convolution $f_{1} * f_{2}$ if $f_{1} \in \mathcal{W}_{H}^{-}(z)$ and $f_{2} \in \mathcal{W}_{H}^{+}(\phi)$.

Theorem 3.1. Let $f_{1} \in \mathcal{W}_{H}^{-}(z)$ and $f_{2} \in \mathcal{W}_{H}^{+}(\phi)$. Then

(i) $f_{1} * f_{2} \in \mathcal{W}_{H}^{+}\left(h_{1} * \phi\right)$;

(ii) Further, if the analytic function $h_{1} * \phi$ is univalent and convex in the direction of imaginary axis and $\operatorname{Re}\left(h_{1} * h_{2}\right)^{\prime} /\left(h_{1} * \phi\right)^{\prime}>1 / 2$ in $\mathbb{D}$, then $f_{1} * f_{2} \in S_{H}^{0}$ and is convex in the direction of imaginary axis. 
Proof. Adding the two identities

$$
z=\left(h_{1}-g_{1}\right) *\left(h_{2}-g_{2}\right) \text { and }\left(h_{1}+g_{1}\right) * \phi=\left(h_{1}+g_{1}\right) *\left(h_{2}+g_{2}\right),
$$

we obtain $h_{1} * h_{2}+g_{1} * g_{2}=h_{1} * \phi$. This shows that $f_{1} * f_{2} \in \mathcal{W}_{H}^{+}\left(h_{1} * \phi\right)$. Similar to the proof of Theorem 2.1, it is easy to see that the condition $\operatorname{Re}\left(h_{1} * h_{2}\right)^{\prime} /\left(h_{1} * \phi\right)^{\prime}>1 / 2$ is equivalent to $\left|w_{f_{1} * f_{2}}\right|<1$ in $\mathbb{D}$. By applying Lemma 1.1, it follows that $f_{1} * f_{2}$ is univalent and convex in the direction of imaginary axis.

Taking $\phi(z) \equiv z$ in Theorem 3.1, we have

Corollary 3.2. Let $f_{1} \in \mathcal{W}_{H}^{-}(z)$ and $f_{2} \in \mathcal{W}_{H}^{+}(z)$. Then $f_{1} * f_{2} \in \mathcal{W}_{H}^{+}(z)$ and if $\operatorname{Re}\left(h_{1} *\right.$ $\left.h_{2}\right)^{\prime}>1 / 2$ in $\mathbb{D}$, then $f_{1} * f_{2} \in S_{H}^{0}$ and is convex in the direction of imaginary axis.

Example 3.3. For $n=2,3, \ldots$, let $q_{n}=r_{n}+\bar{s}_{n}$ be the harmonic mappings of $\mathbb{D}$ with $r_{n}=z-z^{n} / n$ and $s_{n}=z^{n} / n$. Then $q_{n} \in \mathcal{W}_{H}^{+}(z)$ are not univalent in $\mathbb{D}$ and

$$
p_{n} * q_{n}=u_{n} * r_{n}+\overline{v_{n} * s_{n}}=z-\frac{z^{n}}{n^{2}}+\frac{z^{n}}{n^{2}} \quad(n=2,3, \ldots)
$$

where $p_{n}=u_{n}+\bar{v}_{n} \in \mathcal{W}_{H}^{-}(z)$ are defined in Example 2.3.

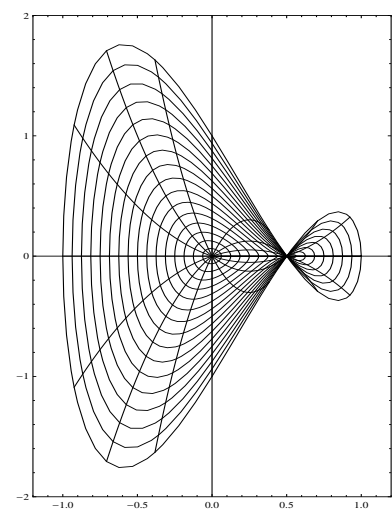

(a) $q_{2}$

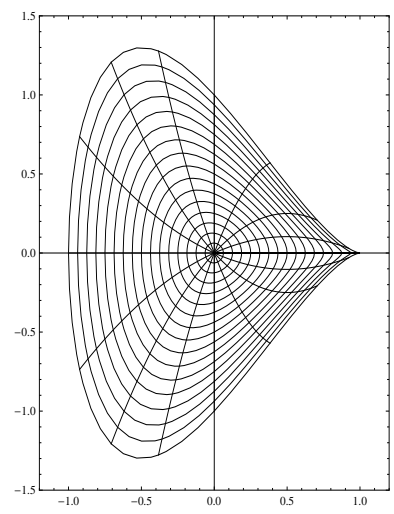

(d) $p_{2} * q_{2}$

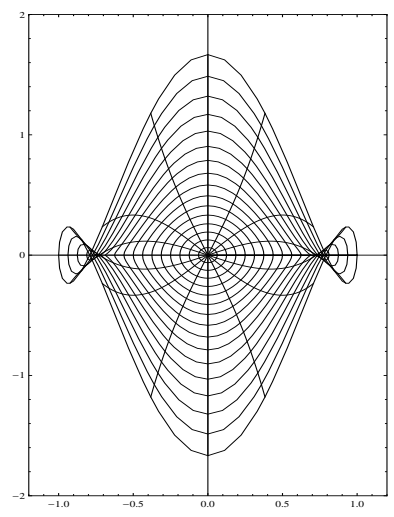

(b) $q_{3}$

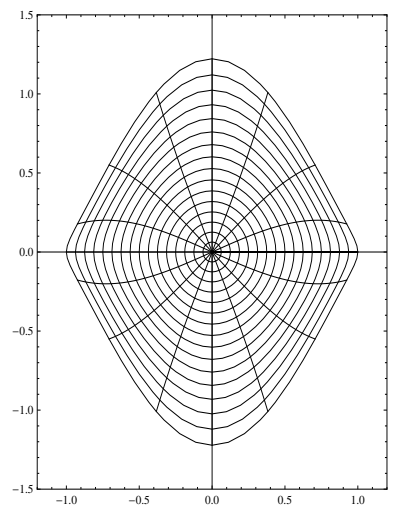

(e) $p_{3} * q_{3}$

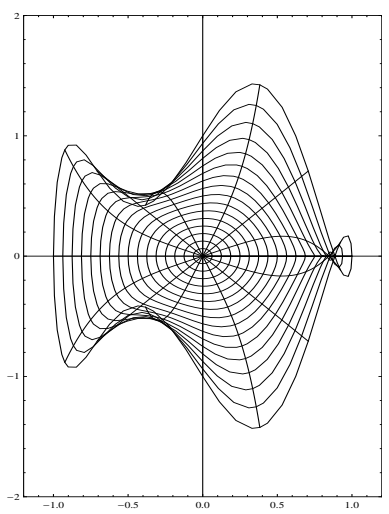

(c) $q_{4}$

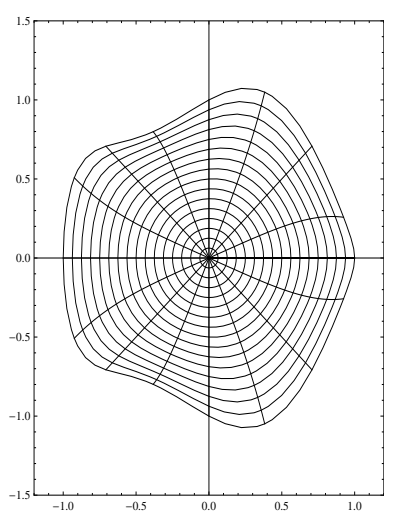

(f) $p_{4} * q_{4}$

Figure 7 . Images of the functions $q_{n}(z)=z-z^{n} / n+\bar{z}^{n} / n$ and the convolutions $p_{n} * q_{n}$ for $n=2,3,4$. 
It is easy to see that $\operatorname{Re}\left(u_{n} * r_{n}\right)^{\prime}>1 / 2$ in $\mathbb{D}$ so that the convolutions $p_{n} * q_{n}(n=2,3, \ldots)$ are univalent and convex in the direction of imaginary axis, by Corollary 3.2. The images of the unit disk under $q_{n}$ and $p_{n} * q_{n}$ are depicted in Figure 7 for $n=2,3,4$.

Example 3.4. For $k=1,2, \ldots$, let $\Psi_{k}=\gamma_{k}+\bar{\delta}_{k}$ be the shears of the identity map in the direction of imaginary axis with dilatation $w_{\Psi_{k}}(z)=z^{k}$. Then $\Psi_{k} \in \mathcal{W}_{H}^{+}(z)$ and

$$
\gamma_{k}(z)=z+\sum_{n=1}^{\infty}(-1)^{n} \frac{z^{n k+1}}{n k+1}, \quad \delta_{k}(z)=\sum_{n=1}^{\infty}(-1)^{n+1} \frac{z^{n k+1}}{n k+1} \quad(z \in \mathbb{D} ; k=1,2, \ldots) .
$$

In particular, $\Psi_{1}(z)=\bar{z}+2 i \arg \{1+z\}$ and $\Psi_{2}(z)=\bar{z}+2 i \operatorname{Im}(\arctan z)$.

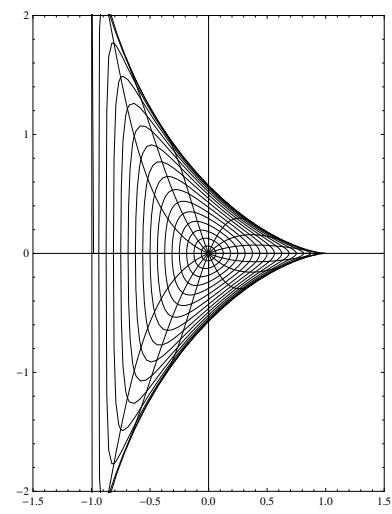

(a) $\Psi_{1}$

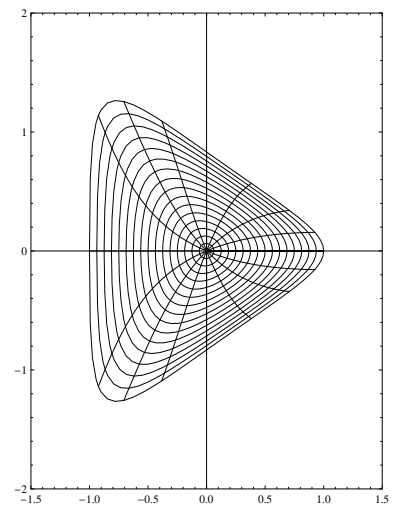

(d) $\Gamma_{1} * \Psi_{1}$

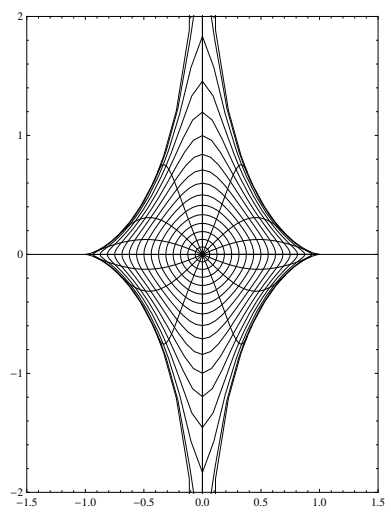

(b) $\Psi_{2}$

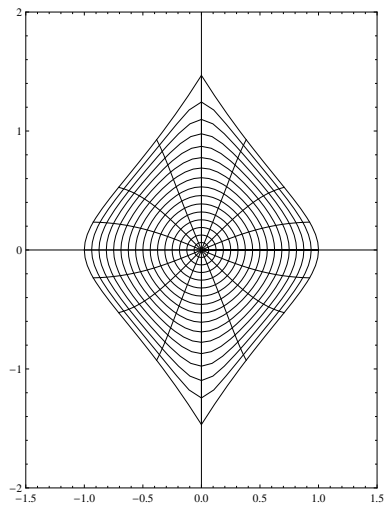

(e) $\Gamma_{2} * \Psi_{2}$

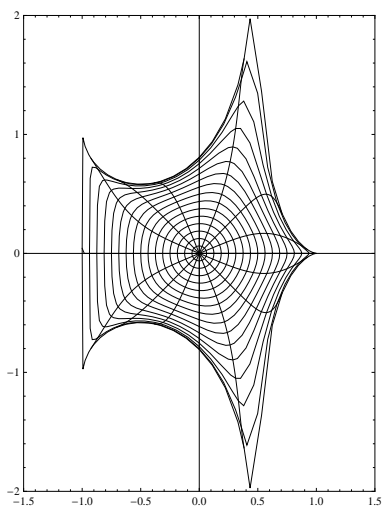

(c) $\Psi_{3}$

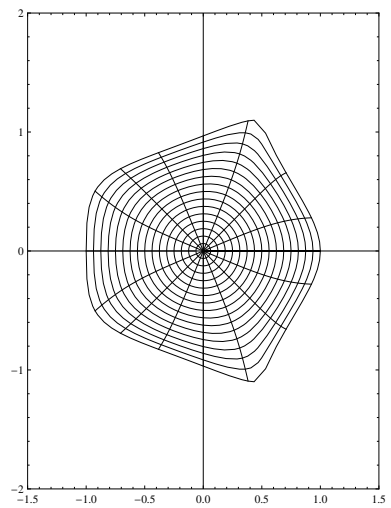

(f) $\Gamma_{3} * \Psi_{3}$

Figure 8. Images of the functions $\Psi_{k}$ and the convolutions $\Gamma_{k} * \Psi_{k}$ for $k=1,2,3$.

Considering the convolutions $\Gamma_{k} * \Psi_{k}(k=1,2, \ldots)$ where $\Gamma_{k}=\mu_{k}+\bar{\nu}_{k}$ are defined in Example 2.5, we see that $\operatorname{Re}\left(z\left(\mu_{k} * \gamma_{k}\right)^{\prime \prime}(z)+\left(\mu_{k} * \gamma_{k}\right)^{\prime}(z)\right)=\operatorname{Re} 1 /\left(1+z^{k}\right)>1 / 2$, for $z \in \mathbb{D}$, which imply that $\operatorname{Re}\left(\mu_{k} * \gamma_{k}\right)^{\prime}>\log 2$ by [13, Theorem 2]. By Corollary [3.2, it follows that the convolutions

$$
\left(\Gamma_{k} * \Psi_{k}\right)(z)=z+\sum_{n=1}^{\infty}(-1)^{n} \frac{z^{n k+1}}{(n k+1)^{2}}+\overline{\sum_{n=1}^{\infty}(-1)^{n+1} \frac{z^{n k+1}}{(n k+1)^{2}}} \quad(z \in \mathbb{D}, k=1,2, \ldots)
$$

are univalent and convex in the direction of imaginary axis (see Figure 8). 
On taking $f_{1} \equiv \Gamma_{1}$ in Theorem 3.1 where $\Gamma_{1} \in \mathcal{W}_{H}^{-}(z)$ is given by (2), we obtain the following corollary. Its proof being similar to the proof of Theorem 2.6 is omitted.

Corollary 3.5. Let $f=h+\bar{g} \in \mathcal{W}_{H}^{+}(\phi)$ with $\operatorname{Re} h(z) / \phi(z)>1 / 2$ for all $z \in \mathbb{D}$. If the analytic function $\mu_{1} * \phi$ is univalent and convex in the direction of imaginary axis, then $\Lambda_{H}^{+}[f] \in \mathcal{S}_{H}^{0}$ and is convex in the direction of imaginary axis, $\Lambda_{H}^{+}$being the positive harmonic Alexander operator defined by (3).

Next, we provide some examples that illustrate Corollary 3.5 for specific choices of $\phi$.

Example 3.6. Consider a non-univalent harmonic function $f=h+\bar{g}$ where $h(z)=z /(1-$ $\left.z^{2}\right)^{2}$ and $g(z)=z^{3} /\left(1-z^{2}\right)^{2}$, belonging to the class $\mathcal{W}_{H}^{+}(\phi), \phi(z)=z\left(1+z^{2}\right) /\left(1-z^{2}\right)^{2}$. Note that $\operatorname{Re} h(z) / \phi(z)=\operatorname{Re} 1 /\left(1+z^{2}\right)>1 / 2$ and $\left(\mu_{1} * \phi\right)(z)=z /\left(1-z^{2}\right)$ is univalent and convex in the direction of imaginary axis. By Corollary [3.5, the convolution

$$
\left(\Gamma_{1} * f\right)(z)=\operatorname{Re} \frac{z}{1-z^{2}}+\frac{1}{2} i \arg \left(\frac{1+z}{1-z}\right), \quad z \in \mathbb{D}
$$

is univalent and convex in the direction of imaginary axis (see Figure 9).

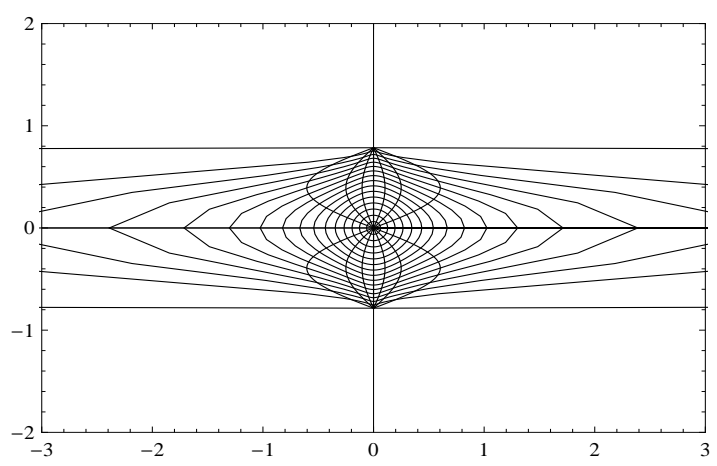

Figure 9. Image of the convolution of $\Gamma_{1}$ with $f(z)=z /\left(1-z^{2}\right)^{2}+\bar{z}^{3} /\left(1-\bar{z}^{2}\right)^{2}$.

Example 3.7. The harmonic half-plane mapping $L=U-\bar{V}, U$ and $V$ are defined in Example 2.12, belongs to $\mathcal{W}_{H}^{+}(z /(1-z))$ and satisfies the hypothesis of Corollary 3.5. Therefore the mapping

$$
\Lambda_{H}^{+}[L](z)=-\log |1-z|+i \operatorname{Im} \frac{z}{1-z}, \quad z \in \mathbb{D}
$$

is univalent and convex in the direction of imaginary axis (see Figure 10).

\section{ACKNOWLEDGEMENTS}

The research work presented here is supported by research fellowship from Council of Scientific and Industrial Research (CSIR), New Delhi and a grant from University of Delhi, Delhi. 


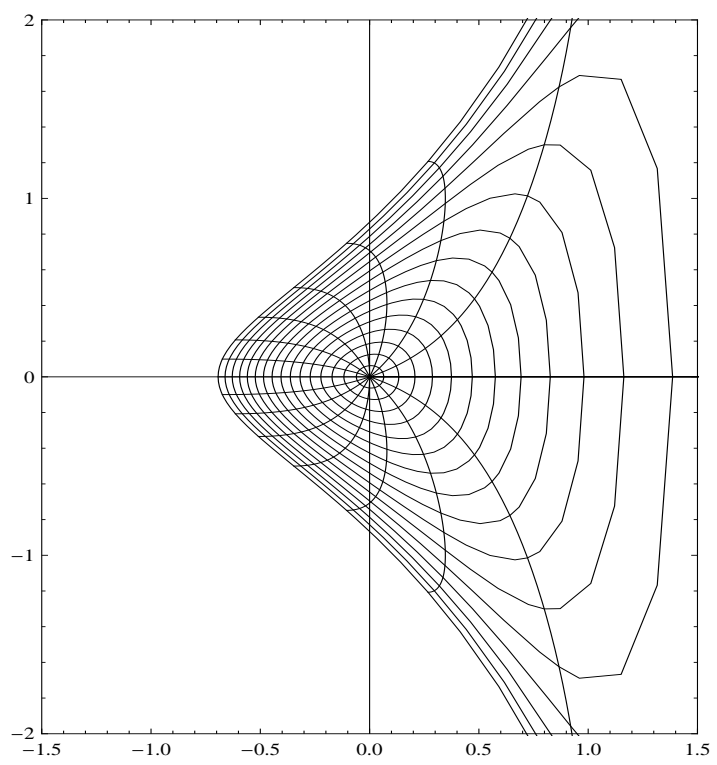

FiguRE 10. Image of the convolution of $\Gamma_{1}$ with the harmonic half-plane mapping $L$.

\section{REFERENCES}

[1] J. Clunie and T. Sheil-Small, Harmonic univalent functions, Ann. Acad. Sci. Fenn. Ser. A I Math. 9 (1984), 3-25.

[2] M. Dorff, Convolutions of planar harmonic convex mappings, Complex Var. Theory Appl. 45 (2001), no. 3, 263-271.

[3] M. Dorff, M. Nowak and M. Wołoszkiewicz, Convolutions of harmonic convex mappings, Complex Var. Elliptic Equ. 57 (2012), no. 5, 489-503.

[4] M. R. Goodloe, Hadamard products of convex harmonic mappings, Complex Var. Theory Appl. 47 (2002), no. 2, 81-92.

[5] S. S. Miller and P. T. Mocanu, Differential subordinations, Monographs and Textbooks in Pure and Applied Mathematics, 225, Dekker, New York, 2000.

[6] P. T. Mocanu, Injectivity conditions in the complex plane, Complex Anal. Oper. Theory 5 (2011), no. 3, 759-766.

[7] S. Nagpal and V. Ravichandran, A subclass of close-to-convex harmonic mappings, Complex Var. Elliptic Equ. (2012), DOI : 10.1080/17476933.2012.727409.

[8] S. Nagpal and V. Ravichandran, Fully starlike and fully convex harmonic mappings of order $\alpha$, Annales Polonici Mathematici, 108 (2013), 85-107.

[9] S. Nagpal and V. Ravichandran, Construction of subclasses of univalent harmonic mappings, arXiv:1209.0075.

[10] S. Nagpal and V. Ravichandran, On a subclass of close-to-convex harmonic mappings, arXiv:1207.3404.

[11] S. Nagpal and V. Ravichandran, A class of harmonic functions with real coefficients defined by convolution, arXiv:1301.2746.

[12] S. Ruscheweyh and L. C. Salinas, On the preservation of direction-convexity and the Goodman-Saff conjecture, Ann. Acad. Sci. Fenn. Ser. A I Math. 14 (1989), no. 1, 63-73.

[13] H. Silverman, A class of bounded starlike functions, Internat. J. Math. Math. Sci. 17 (1994), no. 2, 249-252. 
Department of Mathematics, University of Delhi, Delhi-110 007, India

Department of Mathematics, University of Delhi, Delhi-110 007, India E-mail address: vravi68@gmail.com 